\title{
Left ventricular torsional parameters before and after atrial fibrillation ablation: a velocity vector imaging study
}

Zahra Ojaghi-haghighi ${ }^{1}$, Bahram Mohebbi $^{2}$, Hasan Moladoust ${ }^{3}$, Majid Haghjoo ${ }^{4}$, Azin Alizadehasl ${ }^{5}$, Maryam Esmaeilzadeh ${ }^{6}$, Sevil Aghapour ${ }^{7}$, Hooman Bakhshandeh $^{8}$, Maryam Ardeshiri $^{9}$, Masoumeh Hamidian ${ }^{10}$

${ }^{1}$ Professor, Echocardiography Research Center, Rajaie Cardiovascular Medical \& Research Center, Iran University of Medical Sciences, Tehran, Iran

${ }^{2}$ M.D., Assistant Professor, Interventional Cardiologist, Department of Cardiology, Cardiovascular Intervention Research Center, Rajaie Cardiovascular Medical and Research Center, Faculty of Medicine, Iran University of Medical Sciences, Tehran, Iran

${ }^{3}$ Ph.D., Associate Professor, Healthy Heart Research Center, Guilan University of Medical Sciences, Rasht, Iran

${ }^{4}$ M.D., FESC, FACC, Associate Professor, Cardiac Electrophysiology Research Center, Rajaie Cardiovascular Medical and Research Center, Faculty of Medicine, Iran University of Medical Sciences, Tehran, Iran

5 M.D., Associate Professor, Echocardiologist, Department of Cardiology, Department of Cardiology, Echocardiography Research Center, Rajaie Cardiovascular Medical and Research Center, Faculty of Medicine, Iran University of Medical Sciences, Tehran, Iran

${ }^{6}$ M.D., FACC, FCAPSC, Associate Professor, Echocardiologist, Department of Cardiology, Echocardiography Research Center, Rajaie Cardiovascular Medical and Research Center, Faculty of Medicine, Iran University of Medical Sciences, Tehran, Iran

${ }^{7}$ M.D., Rajaie Cardiovascular Medical and Research Center, Iran University of Medical Sciences, Tehran, Iran

8 M.D., Ph.D., Associate Professor, Epidemiologist, Cardiovascular Intervention Research Center, Rajaie Cardiovascular Medical and Research Center, Faculty of Medicine, Iran University of Medical Sciences, Tehran, Iran

${ }^{9}$ M.D., Assistant Professor of Endocrinology and Metabolism, Rajaie Cardiovascular Medical and Research Center, Iran University of Medical Sciences, Tehran, Iran

${ }^{10}$ B.Sc. of Nursing, Rajaie Cardiovascular Medical and Research Center, Iran University of Medical Sciences, Tehran, Iran

\section{Type of article: Original}

\begin{abstract}
Background and aim: Effects of atrial fibrillation (AF) and its ablative treatment on LV torsion have not yet been fully investigated. This study aimed to examine whether AF patterns of LV contraction and its ablative correction can exert a significant impact on LV torsion by velocity vector imaging (VVI).

Methods: This case-control study conducted in Rajaie Cardiovascular, Medical and Research Center between October 2012 and June 2013. Study participants were 30 consecutive patients with symptomatic paroxysmal AF who met the inclusion criteria. The control group included 24 healthy participants with no history of cardiovascular disease. All individuals were in sinus rhythm at the time of echocardiography before and after the ablation procedure. Two-dimensional (2D) and Doppler echocardiography on a commercially available ultrasound system was performed for all the patients. Scanning was done by a wide-band ultrasound transducer with the frequency range between 2.5-3.5 MHz. The two short-axis views at basal and apical levels were
\end{abstract}

\section{Corresponding author:}

Assistant Professor Dr. Bahram Mohebbi, Department of Cardiology, Cardiovascular Intervention Research Center, Rajaie Cardiovascular Medical and Research Center, Faculty of Medicine, Iran University of Medical Sciences, Vali-Asr Ave., Niyayesh Blvd., Tehran, Zip Code: 1996911151, Iran.

Tel: +98 2123922072, Fax: +982122055594, Email: roodbar@yahoo.com

Received: May 27, 2017, Accepted: August 23, 2017, Published: September 2017

iThenticate screening: August 23, 2017, English editing: September 13, 2017, Quality control: September 15, 2017

This article has been reviewed / commented by three experts

(C) 2017 The Authors. This is an open access article under the terms of the Creative Commons Attribution-NonCommercialNoDerivs License, which permits use and distribution in any medium, provided the original work is properly cited, the use is non-commercial and no modifications or adaptations are made. 
subsequently processed off-line by VVI XStrain software. In order for data analysis, SPSS 16 utilized using paired and independent $t$-test. $p$-value $\leq 0.05$ was considered significant.

Results: $\mathrm{LV}$ torsion $(\% \mathrm{~cm})$ mean $\pm \mathrm{SD}$ was significantly lower in paroxysmal AF patients before ablation $(0.8 \pm 0.3)$ than the control group $(1.5 \pm 0.4)(\mathrm{p}<0.001)$ and increased significantly after ablation $(1.1 \pm 0.5)$ compared with before ablation $(\mathrm{p}=0.004)$, but still significantly lower than the control group $(\mathrm{p}=0.003)$. LV Twist, twist rate and untwist rate mean \pm SD were significantly lower in paroxysmal AF patients before ablation than the control group and increased significantly after ablation compared with before ablation, but still significantly lower than the control group.

Conclusion: Subclinical LV dysfunction may be detected in paroxysmal AF rhythm by measuring torsional parameters through VVI which improves after AF ablation.

Keywords: Paroxysmal atrial fibrillation, Left ventricle, Torsion, Velocity vector imaging

\section{Introduction}

Left ventricular (LV) torsion results from a wringing or twisting motion of the ventricle around its long axis. It is considered as a main contributor to efficient systole and diastole and is, thus, proposed as an indicator for cardiac disease $(1,2)$. Recently, the assessment of LV torsion has been used as a promising tool to quantify LV function, and it is believed that this parameter may be useful in early detection of cardiac disease and clinical decision making $(3,4)$. Cardiovascular magnetic resonance (CMR) tissue tagging is currently the gold standard technique for evaluating LV rotational mechanics (5). But recently; speckle-tracking echocardiography has been applied as a simple and direct method in this regard. Using this quantitative technique, which is now validated with tagged magnetic resonance, has improved torsion-related studies (6). Velocity-vector imaging (VVI) is a new angle independent, two-dimensional echocardiographic method that can be employed to determine LV rotational motion and muscle displacement based on feature tracking performed with Fourier techniques in ultrasound and CMR images $(7,8)$. Atrial fibrillation (AF) is the most common cardiac arrhythmia, and results from electrical and mechanical remodeling even in the absence of structural heart disease. It presents with a wide spectrum of symptoms and severities which need specific approaches to management (9). Pulmonary vein ablation is one choice treatment that achieves the highest cure rate in patients with paroxysmal AF. Some studies have reported that LV torsion is altered under specific physiological and pathological conditions, including normal aging, hypertension, cardiac hypertrophy, cardiomyopathies, ischemia and diabetes (10-16). However, the effects of AF and its ablative treatment on LV rotation and torsion have not yet been fully investigated. This study aimed to examine whether AF patterns of LV contraction and its ablative correction can exert a significant impact on LV torsion by velocity vector imaging (VVI).

\section{Material and Methods \\ 2.1. Study Population}

This case-control study was conducted in Rajaie Cardiovascular, Medical and Research Center between October 2012 and June 2013. The study subjects were 30 consecutive patients with symptomatic paroxysmal AF, who were candidate for ablation referees to Rajaie Cardiovascular, Medical and Research Center. All the patients had normal ejection fraction $(\mathrm{EF}>55 \%)$. Patients with congestive heart failure, valvular disease, ischemic heart disease, left atrial diameter greater than $55 \mathrm{~mm}$ and history of previous ablation procedure were excluded. None of the recruited patients, therefore, had AF due to an underlying structural heart problem. The control group included 24 healthy participants selected from volunteer family members who had no history of cardiovascular disease. All individuals were in sinus rhythm at the time of echocardiography before and after the ablation procedure. Heart rates were similar in control subjects and patients before and after the procedure. Echo studies were performed approximately 24 hours before and after the procedure. Written informed consent was signed by all subjects prior to participation in the study. The study protocol was approved by the institutional review board and local ethics committee.

\subsection{Ablation Procedure}

The patients were kept on continuous oral anticoagulation with intra-procedural INR levels of 2.0-3.0. Ablation procedures were performed under conscious sedation and using a three-dimensional (3D) mapping system for anatomy and catheter visualization (NavX, St. Jude Medical, St. Paul, MN, USA). The individual left atrial (LA) anatomy, as segmented from the previous CT scan, was displayed during the procedure and fused with the reconstructed anatomy in the 3D mapping system if considered appropriate. For pulmonary vein isolation (PVI), a 10 - polar catheter was placed in the coronary sinus (IBI ${ }^{\mathrm{TM}}$, St. Jude Medical, St. Paul, MN, USA), and the LA was accessed via a single trans-septal puncture or via an open foramen oval. After the placement of the electrode catheters within the LA, heparin was given to maintain an activated clotting time of between 300-350 seconds. PVI 
was performed using an irrigated tip ablation catheter (Therapy Cool Path ${ }^{\mathrm{TM}}$, St. Jude Medical, St. Paul, MN, USA). Irrigated radiofrequency $(\mathrm{RF})$ energy with a flow rate of $17 \mathrm{ml} / \mathrm{min}$ was delivered with a maximum temperature of 43 ${ }^{\circ} \mathrm{C}$ and maximum power of $20-25 \mathrm{~W}$ until electric PV isolation confirmed with the circular mapping catheter had been achieved.

\subsection{Echocardiography}

Two-dimensional (2D) and Doppler echocardiography on a commercially available ultrasound system (MyLab60, Esaote Bio., Genova-Italy) was performed for all the patients. Scanning was done by a wide-band ultrasound transducer with the frequency range between 2.5-3.5 MHz (PA230E Esaote Bio., Genova-Italy). LV parasternal short-axis views were obtained at apical and basal levels (mean frame rate $=55.3 \pm 7.0 \mathrm{fps}$ ). The proper basal shortaxis level was defined as that containing the mitral valve and the proper apical short-axis level was defined as that containing the LV cavity with no visible papillary muscles and adjacent RV. The LV cross-section was made as circular as possible. A further cine of apical four-chamber and two-chamber views were stored in order to use modified Biplane Simpson's method to calculate LV ejection fraction (LVEF). End-diastolic LV longitudinal diameter in apical four-chamber view was used to measure LV length. A parasternal long-axis view by M-mode was obtained to measure LV end-diastolic and systolic dimensions and interventricular septal thickness.

\subsection{Velocity Vector Imaging (VVI) and Off-Line Analysis}

The two short-axis views at basal and apical were subsequently processed off-line by using customized software (VVI XStrain software, Esaote Bio, Genova-Italy). For the initial position of the tracking points based on the American Society Echocardiography's (ASE) segmentation of the heart, in the end-systolic frame, the aided heart segmentation (AHS) mode was utilized to insert well equal-spaced endocardial tracking points for each $2 \mathrm{D}$ shortaxis view (17). The software then applied a sequence of processing steps to track the motion of the segments in the other frames automatically. The tissue velocity is displayed as a vector projected on each of the 2D short-axis levels where the vector shows the direction and velocity of the myocardial movement and the rotation of the LV segments around the LV central axis were calculated separately. The data of all the sample regions' tracking were transferred to a spreadsheet Excel program for LV average rotation and rotational velocity calculation at each short-axis level. Basal and apical rotations were calculated as average angular displacement of six myocardial segments. Counterclockwise LV apical rotation and torsion as viewed from the apex were expressed as positive values, and clockwise LV rotation was expressed as negative value for the basal level. LV twist (degrees) is defined as the instantaneous difference between apical rotation and basal rotation. LV twist was calculated as follows (18, 19): LV Twist $(\mathrm{t})=$ Apical LV rotation $(\mathrm{t})$ - Basal LV rotation( $\mathrm{t})$. Also, LV twist rate (degrees/s) was calculated as follows: LV Twist $\operatorname{rate}(\mathrm{t})=$ Apical $\operatorname{Vrot}(\mathrm{t})-\operatorname{Basal} \operatorname{Vrot}(\mathrm{t})$; where Apical $\operatorname{Vrot}(\mathrm{t})$ and Basal $\operatorname{Vrot}(\mathrm{t})$ are the rotational velocity (degree/s) at the apical and basal levels, respectively. Figure 1 shows LV twist and untwist and Figure 2 demonstrates LV twisting rate and untwisting rate throughout one cardiac cycle. LV peak apical and basal rotations, peak systolic twist, peak twisting rate and peak untwisting rate were measured as is demonstrated in Figures 1 and 2. LV torsion was defined as normalized twist to LV end-diastolic longitudinal length. For all measurements, the average derived from 3 consecutive cardiac cycles were finally used.
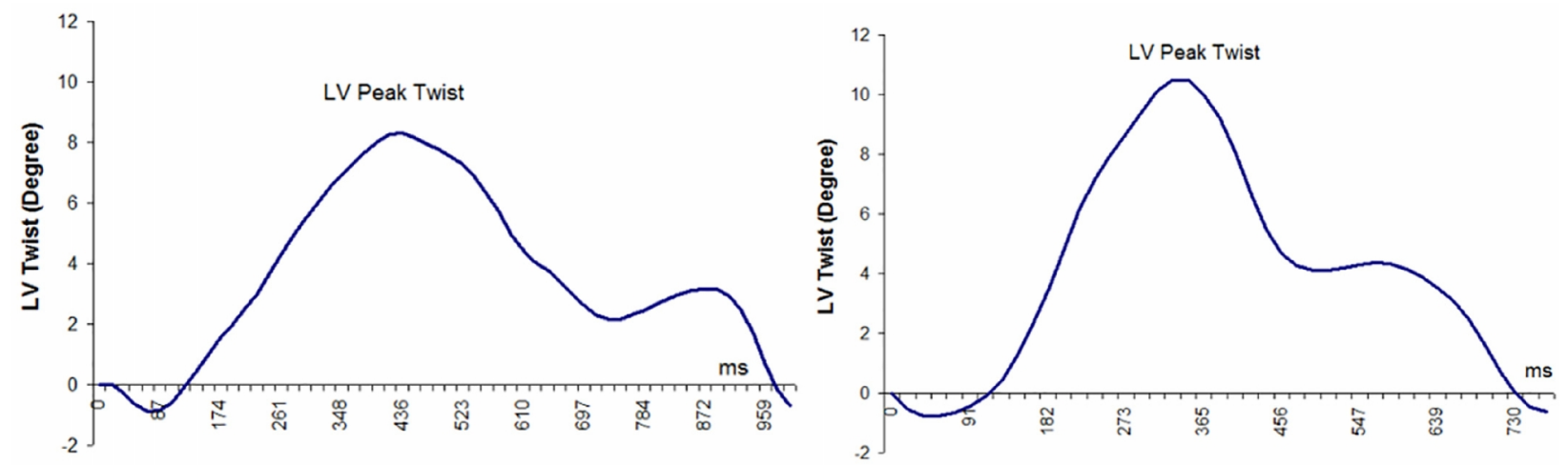

Figure 1. LV twist throughout one cardiac cycle in an AF patient. Left, before and right, after ablation 

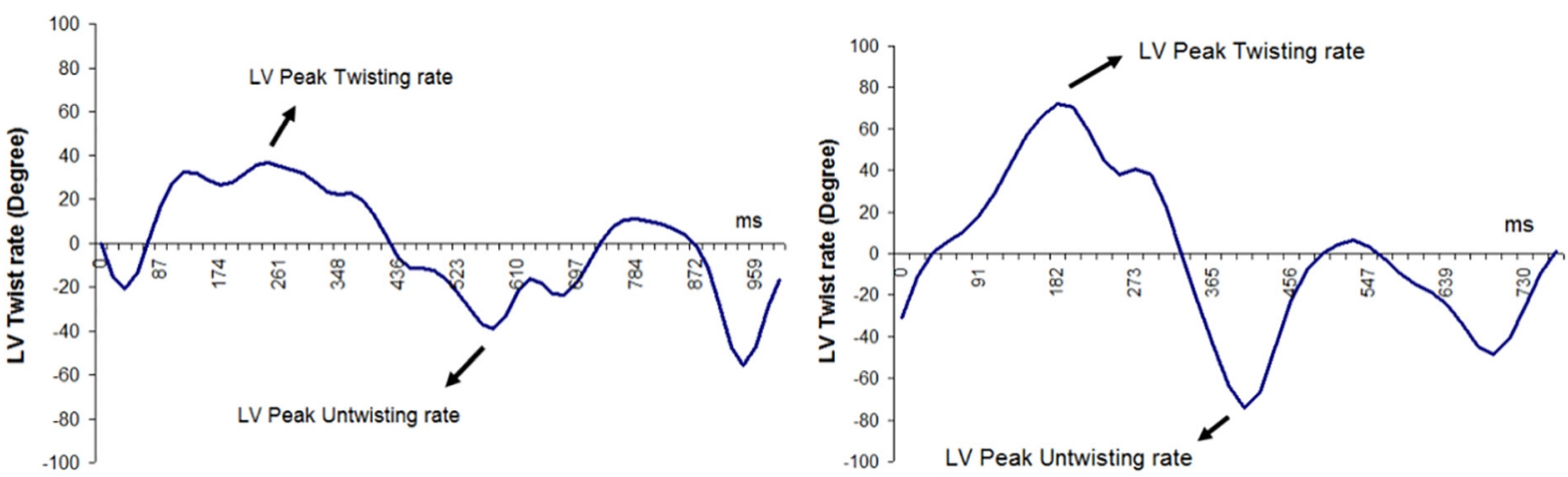

Figure 2. LV twisting and untwisting rate throughout one cardiac cycle in an AF patient. Left, before and right, after ablation

\subsection{Statistical Analysis}

All the continuous variables were tested for normal distribution via Kolmogorov-Smirnov's (K-S) test and are presented as mean \pm standard deviation (SD). The results were compared using paired (before and after ablation) and independent-samples t-test (patients and control). p-value less than or equal to 0.05 was considered the level of statistical significance. Intra-observer variabilities were not different between measurements. All the statistical analyses were performed using SPSS version 16 (SPSS Inc. Chicago, IL, USA).

\section{Results}

\subsection{Clinical and Echocardiographic Characteristics}

The present study evaluated 30 patients and 24 healthy subjects. Three patients were excluded due to their poor acoustic windows, leaving 27 patients (15 males and 12 females; Mean age: $45 \pm 7$ years) and 24 healthy subjects (13 males and 11 females; Mean age: $43 \pm 7$ years). After AF ablation, all the patients had normal sinus rhythm. The echo findings of the subjects are represented in Table 1. There was no significant difference between the patients and the controls in demographic data. None of the patients experienced an acute complication.

\subsection{Torsion Parameters}

LV torsion parameters are shown in Table 2. The paroxysmal AF patients had significantly lower LV apical counterclockwise rotation and basal LV clockwise rotation than had the controls $(\mathrm{p}<0.05)$. Furthermore, LV twist, twist rate and LV torsion were lower in the paroxysmal AF patients. After ablation, however, there was a significant increase in the counter-clockwise rotation of the apex as compared with the prior ablation values $(p<0.05)$; such an increase was not noted in the clockwise rotation of the base $(\mathrm{p}>0.05)$. A significant increase was also noted while comparing pre- and post-ablation LV twist and twist rate. Moreover, LV torsion increased after the treatment. However, there was still a statistically significant difference between the post-ablation values and the amount measured in the controls $(\mathrm{p}<0.05)$. Figures 1 and 2 show LV twist and untwisting rate profiles through one cardiac cycle in a patient before and after ablation. The inter-observer variability for LV twist, torsion, twisting rate and untwisting rate by two independent observers (in 15 randomly selected stored clips) were both blinded to each other's reading and were $7 \%, 8 \%, 10 \%$ and $9 \%$, respectively. The intra-observer variabilities (were performed two weeks after the original analysis) for LV twist, torsion, twisting rate and untwisting rate were $5 \%, 6 \%, 7 \%$ and $7 \%$, respectively.

Table1. The echo findings of the study groups

\begin{tabular}{|l|l|l|l|}
\hline Variables & Before Ablation & After Ablation & Control \\
\hline Left ventricular ejection fraction (\%) & $58 \pm 2$ & $59 \pm 1$ & $60 \pm 2$ \\
\hline left atrial diameter (cm) & $3.2 \pm 0.3$ & $3.3 \pm 0.2$ & $3.3 \pm 0.3$ \\
\hline Left ventricle length (cm) & $7.40 \pm 0.67$ & $7.37 \pm 0.71$ & $7.39 \pm 0.64$ \\
\hline Left ventricular end-diastolic dimension (cm) & $4.7 \pm 0.3$ & $4.6 \pm 0.2$ & $4.5 \pm 0.2$ \\
\hline Interventricular septal thickness (cm) & $0.78 \pm 0.11$ & $0.77 \pm 0.12$ & $0.76 \pm 0.12$ \\
\hline
\end{tabular}


Table 2. LV torsional parameters in the study groups

\begin{tabular}{|l|l|l|l|l|l|l|}
\hline \multirow{2}{*}{$\begin{array}{l}\text { Variables } \\
(\text { Mean } \pm \text { SD) }\end{array}$} & Groups & $\begin{array}{l}\text { Before } \\
\text { ablation }\end{array}$ & $\begin{array}{l}\text { After } \\
\text { ablation }\end{array}$ & $\begin{array}{l}\text { Patients before } \\
\text { ablation vs. } \\
\text { Control* }\end{array}$ & $\begin{array}{l}\text { Patients before } \\
\text { vs. after } \\
\text { ablation** }\end{array}$ & $\begin{array}{l}\text { Patients after } \\
\text { ablation vs . } \\
\text { Control* }\end{array}$ \\
\hline Apical rotation (degree) & $7.1 \pm 2.2$ & $3.6 \pm 1.7$ & $5.1 \pm 2.7$ & $<0.001$ & 0.010 & 0.011 \\
\hline Basal rotation (degree) & $-5.2 \pm 1.7$ & $-3.4 \pm 1.9$ & $-4.0 \pm 2.4$ & 0.001 & 0.215 & 0.047 \\
\hline Left ventricular twist $($ degree) & $11.1 \pm 2.5$ & $5.9 \pm 2.4$ & $8.0 \pm 3.4$ & $<0.001$ & 0.005 & 0.001 \\
\hline Left ventricular twist rate $(\% / \mathrm{s})$ & $75.9 \pm 17.3$ & $48.5 \pm 13.5$ & $61.4 \pm 18.5$ & $<0.001$ & 0.012 & 0.006 \\
\hline Left ventricular untwist rate $(\% / \mathrm{s})$ & $-73.8 \pm 24.5$ & $-45.4 \pm 15.0$ & $-61.2 \pm 24.9$ & $<0.001$ & 0.015 & $<0.001$ \\
\hline Left ventricular torsion $(\% / \mathrm{cm})$ & $1.5 \pm 0.4$ & $0.8 \pm 0.3$ & $1.1 \pm 0.5$ & $<0.001$ & 0.004 & 0.003 \\
\hline
\end{tabular}

*Independent samples t-test, **paired-samples t-test

\section{Discussion}

Since there were few studies related to the aim of this study, discussion was focused on the few published studies relatively close to the aim of this study. LV torsion may appear as a more sensitive parameter to diagnose LV dysfunction compared with some traditional echocardiographic variables, so LV torsion may be considered as an important diagnostic factor for LV dysfunction (20). The present study was designed to investigate the LV torsional parameters, as assessed by VVI, in paroxysmal AF patients before and after ablation therapy. We measured LV torsion by dividing the twist by LV length. To eliminate the effect of the confounding factors, only paroxysmal AF patients with normal heart rate and normal ejection fraction were recruited; whereas we reported an approximate LV twist value of $11.1^{\circ} \pm 2.5^{\circ}$ in our controls. However, different values for LV torsion in normal subjects have been reported in the literature. Peak LV twist calculated in a Sandstede et al. (21) study was $14.5^{\circ} \pm 3.7^{\circ}$. Data closer to our findings were reported by Young et al. (22) between $12^{\circ}$ and $14^{\circ}$, and much lower values were reported by Stuber et al. (23) and Nagel et al. (13). These differences may be due to the different definitions applied for the apical slice, technique and image settings, including focus, sector width, frame rate, gain, etc. Silent arrhythmia is prevalent in AF patients; the condition is found in the clinical examination of about $20 \%$ of patients (24). There are reports of silent arrhythmia in $50 \%$ of asymptomatic $\mathrm{AF}$ patients using transtelephonic arrhythmia monitoring or implantable monitoring devices $(24,25)$. In this study, findings revealed a significant reduction in pre-AF ablation $\mathrm{LV}$ basal rotation (to $-3.4 \pm 1.9^{\circ}$ ), $\mathrm{LV}$ apical rotation (to $3.6^{\circ} \pm 1.7^{\circ}$ ) and $\mathrm{LV}$ twist value (to $5.9^{\circ} \pm 2.4^{\circ}$ ) in patients with paroxysmal AF compared with healthy control subjects despite normal ejection fraction in both groups. It is suggested, changes in LV torsion parameters represent subclinical LV dysfunction in patients with paroxysmal AF even when they are in sinus rhythm. According to our results, apical and basal rotation and torsion values were remarkably lower in the paroxysmal AF patients than those in the controls. Considering the fact that all our patients had normal EF values, it could be concluded that torsion values are affected earlier than EF values. We suggested that changes in LV torsion parameters represent subclinical LV dysfunction in patients with paroxysmal AF even when they are in sinus rhythm. Different studies showed the value of torsional parameters as a marker of subclinical LV dysfunction in valvular heart disease, as Sandstede et al. (21) assessed LV torsion in patients with aortic stenosis before and after surgical valve replacement. To our knowledge, there is no study in the existing literature to date to measure LV twist/torsion during AF. Our findings similarly indicated that LV torsion was a sensitive marker for early detection of $\mathrm{LV}$ dysfunction during $\mathrm{AF}$, and might be used to determine the proper timing of ablation.

Recently, there has been an increasing interest in the use of catheter ablation, as a safe and efficient alternative to drug therapy, for the treatment of AF patients. In our study, LV twist and torsion improved 24 hours after ablation therapy. While the apical counterclockwise rotation values increased during the study, there was no significant increase in the basal clockwise rotation values. Consequently, the effects of ablation therapy were more frequently seen in the apex than the base. LV twist and torsion increased 24 hours following ablation. Whether the reason is an improved atrial contraction or an improved intrinsic LV myocardial function, or as a result of biomarkers, is an unknown and further studies need to be undertaken to find out the answer. The lack of complete improvement in LV twist and torsion, by comparison with the control group, in this study could be due to the fact that these values were measured shortly after ablation. We suggest that torsion be measured in time periods longer than 24 hours after ablation. This rapid change in torsion values also may suggest torsional parameters as a monitoring marker for post ablation AF recurrence. As Hindricks et al. (26) studied the relevance of asymptomatic arrhythmia recurrence after ablation and showed that asymptomatic episodes might occur after catheter ablation. They suggested that if these patients had been followed only based on the symptoms, it might have led to underestimating the rate of recurrences and as a result, recommending long-term Holter monitoring as an objective measure to detect asymptomatic AF 
recurrences. In this study, our findings suggest that LV torsion might be used for post-ablation monitoring in patients with $\mathrm{AF}$, as any reduction or even lack of improvement in LV torsion after AF ablation might be related to the recurrence of AF following ablation in a structurally normal heart. Since image quality is an important determinant in VVI method, we excluded 3 patients due to poor image quality. Second, peak apical rotation depends markedly on the level of the scanned apex which was specifically addressed by Van Dalen et al. (27). In this study, we tried to obtain the closest plane to the apical obliteration and this limitation can equally influence in a patient at before and after AF ablation apical views. Further investigation with larger number of patients is required to confirm these results. CMR tissue tagging and 3D echocardiography can improve the accuracy of the assessment of LV torsional parameters, but they are not widely available.

\section{Conclusions}

AF significantly affects LV twist and untwisting rate compared to controls. Moreover, AF ablation leads to significant improvement of apical rotation compared to basal rotation, but the extent of this change significantly differs from controls, concluding AF may result in subclinical LV dysfunction in the presence of normal EF. These data show that apical rotation, twisting and untwisting rate are sensitive markers to detect these abnormalities. Post $\mathrm{AF}$ ablation reversible subclinical LV dysfunction may be detected in paroxysmal AF rhythm by measuring torsional parameters through VVI study. LV torsion could be used for post-ablation monitoring in patients with paroxysmal $\mathrm{AF}$, as a decline and lack of improvement in $\mathrm{LV}$ torsion after $\mathrm{AF}$ ablation might be related to the recurrence of $\mathrm{AF}$ following ablation.

\section{Acknowledgments:}

We would like to thank all participants who dedicated their own time to study participation. We are grateful to Rajaie Cardiovascular, Medical and Research Center which allowed us to conduct this study.

\section{Conflict of Interest:}

There is no conflict of interest to be declared.

Authors' contributions:

All authors contributed to this project and article equally. All authors read and approved the final manuscript.

\section{References:}

1) Arts T, Reneman RS, Veenstra PC. A model of the mechanics of the left ventricle. Annals of biomedical engineering. 1979; 7(3-4): 299-318. doi: 10.1007/BF02364118. PMID: 547767.

2) Notomi Y, Popović ZB, Yamada H, Wallick DW, Martin MG, Oryszak SJ, et al. Ventricular untwisting: a temporal link between left ventricular relaxation and suction. Am J Physiol Heart Circ Physiol. 2008; 294(1): H505-13. doi:10.1152/ajpheart.00975.2007. PMID: 18032523.

3) Hansen DE, Daughters G, Alderman E, Stinson E, Baldwin J, Miller DC. Effect of acute human cardiac allograft rejection on left ventricular systolic torsion and diastolic recoil measured by intramyocardial markers. Circulation. 1987; 76(5): 998-1008. doi: 10.1161/01.CIR.76.5.998. PMID: 3311453.

4) Shirakabe A, Ikeda Y, Sciarretta S, Zablocki DK, Sadoshima J. Aging and Autophagy in the Heart. Circ Res. 2016; 118(10): 1563-76. doi: 10.1161/CIRCRESAHA.116.307474. PMID: 27174950.

5) Ho SY. Anatomy and myoarchitecture of the left ventricular wall in normal and in disease. Eur $J$ Echocardiogr. 2009 ; 10(8): iii3-7. doi: 10.1093/ejechocard/jep159. PMID: 19889656.

6) Helle-Valle T, Crosby J, Edvardsen T, Lyseggen E, Amundsen BH, Smith HJ, et al. New noninvasive method for assessment of left ventricular rotation speckle tracking echocardiography. Circulation. 2005; 112(20): 3149-56. doi: 10.1161/CIRCULATIONAHA.104.531558. PMID: 16286606.

7) Pirat B, Khoury DS, Hartley CJ, Tiller L, Rao L, Schulz DG, et al. A novel feature-tracking echocardiographic method for the quantitation of regional myocardial functionValidation in an animal model of ischemia-reperfusion. Journal of the American College of Cardiology. 2008; 51(6): 651-9. doi: 10.1016/j.jacc.2007.10.029. PMID: 18261685.

8) Shaw SM, Fox DJ, Williams SG. The development of left ventricular torsion and its clinical relevance. Int J Cardiol. 2008; 130(3): 319-25. doi: 10.1016/j.ijcard.2008.05.061. PMID: 18678418.

9) Karliner JS, Gault JH, Bouchard RJ, Holzer J. Factors influencing the ejection fraction and the mean rate of circumferential fibre shortening during atrial fibrillation in man. Cardiovascular research. 1974; 8(1): 18 25. PMID: 4819485. 
10) Lumens J, Delhaas $T$, Arts $T$, Cowan BR, Young AA. Impaired subendocardial contractile myofiber function in asymptomatic aged humans, as detected using MRI. Am J Physiol Heart Circ Physiol. 2006; 291(4): H1573-H9. doi: 10.1152/ajpheart.00074.2006. PMID: 16679404.

11) Arts T, Hunter WC, Douglas AS, Muijtjens AM, Corsel JW, Reneman RS. Macroscopic three-dimensional motion patterns of the left ventricle. Adv Exp Med Biol. 1993; 346: 383-92. PMID: 8184778.

12) Bertini M, Delgado V, Nucifora G, Marsan NA, Ng AC, Shanks M, et al. Left ventricular rotational mechanics in patients with coronary artery disease: differences in subendocardial and subepicardial layers. Heart. 2010; 96(21): 1737-43. doi: 10.1136/hrt.2010.197533. PMID: 20956489.

13) Nagel E, Stuber M, Burkhard B, Fischer SE, Scheidegger MB, Boesiger P, et al. Cardiac rotation and relaxation in patients with aortic valve stenosis. European Heart Journal. 2000; 21(7): 582-9. PMID: 10775013.

14) Young AA, Kramer CM, Ferrari VA, Axel L, Reichek N. Three-dimensional left ventricular deformation in hypertrophic cardiomyopathy. Circulation. 1994; 90(2): 854-67. doi: 10.1161/01.CIR.90.2.854. PMID: 8044957.

15) Kanzaki H, Nakatani S, Yamada N, Urayama Si, Miyatake K, Kitakaze M. Impaired systolic torsion in dilated cardiomyopathy: reversal of apical rotation at mid-systole characterized with magnetic resonance tagging method. Basic research in cardiology. 2006; 101(6): 465-70. doi: 10.1007/s00395-006-0603-6. PMID: 16783487.

16) Fonseca CG, Dissanayake AM, Doughty RN, Whalley GA, Gamble GD, Cowan BR, et al. Threedimensional assessment of left ventricular systolic strain in patients with type 2 diabetes mellitus, diastolic dysfunction, and normal ejection fraction. The American journal of cardiology. 2004; 94(11): 1391-5. doi: 10.1016/j.amjcard.2004.07.143. PMID: 15566909.

17) Aminian F, Esmaeilzadeh M, Moladoust H, Maleki M, Shahrzad S, Emkanjoo Z, et al. Does accessory pathway significantly alter left ventricular twist/torsion? A study in Wolff - Parkinson - White syndrome by velocity vector imaging. Echocardiography. 2014; 31(7): 872-8. doi: 10.1111/echo.12470. PMID: 24372902.

18) Esch BT, Warburton DE. Left ventricular torsion and recoil: implications for exercise performance and cardiovascular disease. Journal of Applied Physiology. 2009; 106(2): 362-9. doi: 10.1152/japplphysiol.00144.2008. PMID: 18988768.

19) Rüssel IK, Götte MJ, Bronzwaer JG, Knaapen P, Paulus WJ, van Rossum AC. Left ventricular torsion: an expanding role in the analysis of myocardial dysfunction. JACC: Cardiovascular Imaging. 2009; 2(5): 64855. doi: 10.1016/j.jcmg.2009.03.001. PMID: 19442954.

20) Esch BT, Warburton DER. Left ventricular torsion and recoil: implications for exercise performance and cardiovascular disease. J Appl Physiol. 2009; 106(2): 362-9. doi:10.1152/japplphysiol.00144.2008. PMID: 18988768.

21) Sandstede JJ, Johnson T, Harre K, Beer M, Hofmann S, Pabst T, et al. Cardiac systolic rotation and contraction before and after valve replacement for aortic stenosis: a myocardial tagging study using MR imaging. American Journal of Roentgenology. 2002; 178(4): 953-8. doi: 10.2214/ajr.178.4.1780953.

22) Young AA, Imai $\mathrm{H}$, Chang $\mathrm{CN}$, Axel L. Two-dimensional left ventricular deformation during systole using magnetic resonance imaging with spatial modulation of magnetization. Circulation. 1994; 89(2): 740-52. doi: 10.1161/01.CIR.89.2.740. PMID: 8313563.

23) Stuber M, Scheidegger M, Fischer S, Nagel E, Steinemann F, Hess O, et al. Alterations in the local myocardial motion pattern in patients suffering from pressure overload due to aortic stenosis. Circulation. 1999; 100(4): 361-8. doi: 10.1161/01.CIR.100.4.361. PMID: 10421595.

24) Rho RW, Page RL. Asymptomatic atrial fibrillation. Prog Cardiovasc Dis. 2005 ;48(2): 79-87. doi: 10.1016/j.pcad.2005.06.005. PMID: 16253649.

25) Israel CW, Grönefeld G, Ehrlich JR, Li YG, Hohnloser SH. Long-term risk of recurrent atrial fibrillation as documented by an implantable monitoring deviceimplications for optimal patient care. Journal of the American College of Cardiology. 2004; 43(1): 47-52. doi: 10.1016/j.jacc.2003.08.027. PMID: 14715182.

26) Hindricks G, Piorkowski C, Tanner H, Kobza R, Gerds-Li JH, Carbucicchio C, et al. Perception of Atrial fibrillation before and after radiofrequency catheter ablation relevance of asymptomatic arrhythmia recurrence. Circulation. 2005; 112(3): 307-13. doi: 10.1161/CIRCULATIONAHA.104.518837. PMID: 16009793.

27) van Dalen BM, Vletter WB, Soliman OI, ten Cate FJ, Geleijnse ML. Importance of transducer position in the assessment of apical rotation by speckle tracking echocardiography. Journal of the American Society of Echocardiography. 2008; 21(8): 895-8. doi: 10.1016/j.echo.2008.02.001. PMID: 18356018. 